\title{
Prévision de Défaillance Des entreprises : Apport des Réseaux de Neurones Artificiels
}

\author{
Siham LOTFI ${ }^{1}$ and Hicham MESK ${ }^{2}$ \\ ${ }^{I}$ Enseignante Chercheure à l'Université Internationale de Casablanca, \\ Doctorante : Laboratoire Business Intelligence, Gouvernance des Organisations et Finance, \\ Faculté des Sciences Juridiques, Économiques et Sociales \\ Université Hassan II, Casablanca, Maroc \\ ${ }^{2}$ Professeur de l'enseignement supérieur \\ Laboratoire Business Intelligence, Gouvernance des Organisations et Finance, \\ Faculté des Sciences Juridiques, Économiques et Sociales \\ Université Hassan II, Casablanca, Maroc
}

\begin{abstract}
Résumé : Le principal problème auquel font face les banques lors de la décision de l'octroi de crédit est leur incapacité à déterminer avec certitude si le client va honorer ou non ses engagements. Depuis toujours, cette décision de l'octroi du crédit repose sur l'évaluation préalable de l'agent de crédit. En effet, une détection précoce des difficultés de l'entreprise se fait à l'aide des outils de prévision du risque de défaillance qui s'appuient tous sur l'analyse du passé pour prédire l'avenir de l'entreprise. Cette analyse repose essentiellement sur l'exploitation des états de synthèse de l'entreprise qui restent une source d'informations incontournable pour la détection des difficultés des entreprises. Parmi ces méthodes de prévision, on trouve les réseaux de neurones artificiels. Cette technique est utilisée dans de nombreuses disciplines notamment la médecine, le marketing, la finance et constitue une alternative intéressante aux techniques statistiques traditionnelles pour le traitement des données comme (la régression logistique, analyse discriminante, etc..).
\end{abstract}

Mots-clés : Prédiction, Défaillance, Risque de crédit, Évaluation, Réseaux de neurones artificiels, Intelligence artificielle.

\section{INTRODUCTION}

La notion de défaillance revêt une diversité assez large des terminologies qui permettent de qualifier une entreprise de défaillante. Cette diversité s'explique par le fait que la défaillance est la résultante de plusieurs causes qu'on pourrait classer en deux catégories, des causes micro-économiques dont l'origine est interne à l'entreprise, notamment les différents aspects de l'organisation et des causes macro-économiques liées à la conjoncture économique sectorielle nationale.

En effet, la gestion du risque de défaillance ne peut être pratiquée d'une manière efficace sans la connaissance du marché du crédit ainsi que les différents déterminants relatifs au secteur bancaire qui peuvent apprécier ce risque.

Dans leur article, (Yoser Gadhoum et al 2007), soulignent que l'évaluation du risque de crédit représente une phase primordiale de la gestion du risque de crédit car elle dépend de la performance du crédit octroyé au 
client. La banque doit alors, déterminer le plus adéquatement possible le risque réel de l'emprunteur afin de protéger sa trésorerie, anticiper et prévenir les impayés.

De ce fait, les établissements bancaires sont donc très intéressés à développer des modèles d'analyse, de mesure et d'évaluation du risque de crédit plus précis afin d'apprécier le rendement des prêts accordés.

Parmi ces méthodes de prédiction, on peut citer le crédit Scoring, cette méthode implique l'exploitation des différentes techniques statistiques pour aboutir à une fonction score basée sur les caractéristiques de l'emprunteur et qui aide à la prise de décision dans l'octroi de crédit.

Dans la pratique, on trouve une variété de techniques allant des méthodes conventionnelles telle la méthode de régression linéaire multiple, analyse probit, régression logistique, analyse discriminante ou bien des techniques avancées comme l'algorithmique, les systèmes d'expert, (Hand \& Henly, 1997).

Dans la littérature financière, d'autres méthodes ont été récemment développées comme les modèles basés sur l'intelligence artificielle. Parmi ces méthodes, on trouve la technique des réseaux de neurones artificiels.

\section{LE CRÉDIT SCORING}

Le crédit scoring est généralement considéré comme une méthode d'évaluation utilisé par les organismes bancaires pour estimer le risque de défaut et mesurer la solvabilité de chaque entreprise et lui classer soit comme une entreprise saine ou une entreprise défaillante. Il impose d'utiliser les différentes techniques statistiques en vue d'obtenir un modèle de scoring basé sur les caractéristiques de l'emprunteur.

Pour (Flaman, 1997), le crédit scoring est le processus d'assignation d'une note (ou score) à un emprunteur potentiel pour estimer la performance future de son prêt ${ }^{1}$.

Le crédit Scoring constitue un ensemble de modèles de décision et les techniques sous- jacentes qui aident dans la décision d'octroi des crédits de consommation (Thomas et al. 2002).

(Bardos, 2001, 2008a, 2008b), souligne que le scoring est une analyse statistique permettant de prédire la qualité d'un emprunteur Cette méthode s'inscrit dans le Data Mining ${ }^{2}$, il est défini par R. Anderson (2007) ${ }^{3}$ comme étant le recours aux modèles statistiques en vue de transformer des données (qualitatives, quantitatives) en indicateurs numériques mesurables à des fins d'aide à la décision d'octroi ou de rejet de crédit.

La technique de scoring permet d'utiliser des modèles statistiques afin de transformer des données (qualitatives, quantitatives) en indicateurs numériques mesurables à des fins d'aide à la décision d'octroi ou de rejet de crédit.

À travers l'historique des données des prêts précédents, le scoring permet d'attribuer une note appelée « score », qui permet de prédire la probabilité de défaut pour les nouveaux crédits.

En effet, la théorie du scoring postule que le passé est le meilleur estimateur du futur. Le processus du crédit scoring peut être résumé comme suit :

${ }^{1}$ Boubacar Diallo, 2006 «UN MODELE DE “CREDIT SCORING” POUR UNE INSTITUTION DE MICROFINANCE AFRICAINE : LE CAS DE NYESIGISO AU MALI ». HAL Id : halshs-00069163

$\mathrm{P}: 4$

${ }^{2}$ Le Data Mining correspond à l'ensemble des techniques reposant aussi bien sur la statistique, l'informatique que l'intelligence artificielle, qui permettent l'extraction de connaissances à partir de bases de données de grande taille

${ }^{3}$ Anderson, R. (2007). "The Credit Scoring Toolkit: Theory and Practice for Retail Credit Risk Management and Decision Automation". New York : Oxford University Press. 


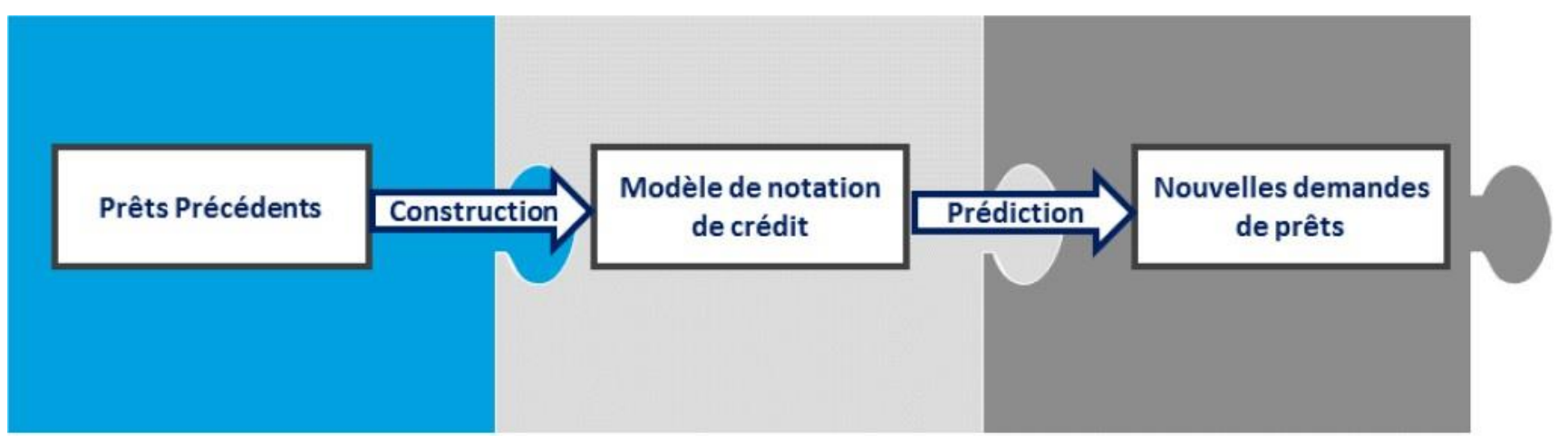

Figure 1. Processus du Crédit Scoring.

Source: Yang Liu, (2001), New Issues in Credit Scoring Application, Work report N 16 , Institut fûr Wirtschaftsinformatik, 2001.

Avec le développement des différents besoins des systèmes de crédit scoring, on assiste à une variété des méthodes d'évaluation du risque de crédit avec un objectif identique qui est l'augmentation de l'efficacité des prises de décision. Parmi ces techniques, on trouve les réseaux de neurones artificiels.

\section{LES RÉSEAUX DE NEURONES ARTIFICIELS}

Parmi les méthodes non paramétriques, on trouve les réseaux de neurones qui occupent une place prépondérante dans le domaine de classification et de prédiction. Cette approche neuronale a récemment attiré 1'attention des chercheurs. Elle s'est développée dès les années 80, pour faire face aux insuffisances de l'analyse discriminante dans la prédiction de la défaillance d'entreprises (Anandarajan, Lee et Anandarajan, 2001). Ils ont été utilisés pour mesurer le risque de défaillance par les ratios financiers, (Leshno et Spector, 1996; Bardos et Zhu, 1997).

Ces modèles neuronaux sont utilisés dans différents domaines comme le marketing, le data mining, la médecine..., (Liu et $\mathrm{Yu}, 2005$ ), mais ils sont moins exploités dans le champ de la prédiction du risque de défaillance.

(Dreyfus et al., 2004), souligne que les réseaux de neurones sont capables d'appréhender tout type de fonction et de réaliser des modèles de prédiction équivalente à ceux produits par des méthodes traditionnelles non linéaires, mais avec moins de paramètres ajustables, ou bien des modèles plus précis à partir d'un même nombre de paramètres.

Les réseaux de neurones ${ }^{4}$ sont inspirés des systèmes neurobiologiques, fabriqués de structures cellulaires artificielles, ils constituent une approche permettant d'aborder les problèmes de perception, de mémoire, d'apprentissage. Ils s'avèrent aussi des alternatives très prometteuses pour contourner certaines insuffisances des méthodes numériques classiques.

Grâce à leur traitement parallèle de l'information et à leurs mécanismes inspirés des cellules nerveuses, ils ont des propriétés émergentes permettant de solutionner des problèmes complexes. Ils sont utilisés en finance, comme ils peuvent être appliqués à d'autres disciplines. En effet, l'utilisation des réseaux neurones dans l'analyse financière a été établie par Malhotra (2002) dans le cadre de la prédiction du phénomène de défaillance.

\footnotetext{
${ }^{4}$ FEKIH Nassima, 2014, L'apport des réseaux de neurones artificiels appliqués au management des risques comme outil de l'audit. Thèse soutenue en 2014, Université Abou Baker Belkaid Tlemcen Faculté des Sciences Economiques, Commerciales et de Gestion.
} 


\section{A. Le fonctionnement des réseaux de neurones artificiels}

Le fonctionnement des RNA ressemble au fonctionnement du cerveau humain, il est composé de millions d'unités, chaque unité ou nœud a une synapse, cette dernière transforme des inputs qui sont en eux même l'output du nœud précédent.

Les neurones d'entrée reçoivent les inputs (les entrées) de l'environnement (La base de données de l'extérieur). Des poids synaptiques s'affectent à chaque entrée, puis, les entrées pondérées sont sommées. Ces résultats se transforment en outputs par une fonction de transfert. Cette dernière peut être sous plusieurs formes (sigmoïde, hyperbolique tangente ou autre).

Le RNA représente un instrument de traitement de l'information, à cet effet la collecte des informations est une étape essentielle qui affecte énormément la performance du réseau et la sincérité et l'utilité des résultats obtenus. Sans information adéquate, fiable et représentative, il est impossible de construire un réseau utile. L'efficacité du réseau dépend toujours de la qualité de la base de données (Zhang, 2003, P20).

Le choix d'un modèle de réseau de neurone optimal repose sur cinq principaux éléments :

- Le type de problème à traiter,

- L'architecture optimale,

- La collecte de la base de données,

- La fonction de transfert,

- L’algorithme d'apprentissage,

Le fonctionnement d'un réseau de neurone passe par trois phases : la phase d'input, la phase de la fonction de transfert et la phase d'apprentissage.

\section{B. La phase d'Input}

Les réseaux reçoivent les informations sur une couche réceptrice contenant des neurones. Ces derniers traitent ces informations avec ou sans l'aide d'une ou plusieurs couches cachées contenant une ou plusieurs neurones produisant un signal ou plusieurs de sortie.

Chaque neurone appartenant soit à la première couche (réceptrice), soit aux couches cachées ou à la couche de sortie est liée aux autres neurones par des connexions (similaires aux synapses du cerveau) auxquelles sont affecté des poids (eux même assimilables aux potentiels synaptiques) ${ }^{5}$.

Les réseaux de neurones, dans le cadre du crédit scoring, permettent de mettre en relation les inputs (la base de données qui est composée des dossiers de crédits) et les outputs (le résultat du crédit : bons payeurs ou mauvais payeurs) sans supposer que cette relation est linéaire.

Le processus de traitement se présente comme suit :

\section{- Une couche d'input}

La couche d'input est composée des neurones qui reçoivent des signaux de l'environnement. Cette couche sert à distribuer les valeurs d'entrées aux neurones des couches supérieures, éventuellement multipliées ou

${ }^{5}$ Thuillier. D (1997), « Principe et applications des réseaux de neurones - deux illustration sur l'habitat au Maroc »; revue région et développement $\mathrm{n}^{\circ}=5$-, page 3 
modifiées d'une façon ou d'une autre. Le nombre de neurones d'entrée dépendent du nombre de variables dans le vecteur d'input.

\section{- Des couches cachées}

Les neurones dans ces couches n'ont aucune interaction directe avec l'environnement. Ils se situent entre la couche d'entrée et la couche de sortie. La détermination du nombre des neurones dans les couches cachées est la tâche la plus difficile dans les réseaux de neurones en général. Il est préférable d'utiliser un nombre réduit de neurones cachés mais il est insuffisant aussi d'utiliser que deux neurones pour modéliser un ensemble de données ${ }^{6}$.

\section{- Une couche d'output}

Les neurones de cette couche émettent des signaux à l'environnement. Elle calcule une somme pondérée de toutes ses entrées. Le nombre des neurones de sortie est rattaché au problème étudié.

La figure ci-dessous montre bien le fonctionnement et l'architecture d'un réseau de neurone. En effet, Chaque entrée est liée à un poids $\mathrm{w}_{\mathrm{i}}$ appelé aussi poids synaptique, ce poids constitue une force de connexion.

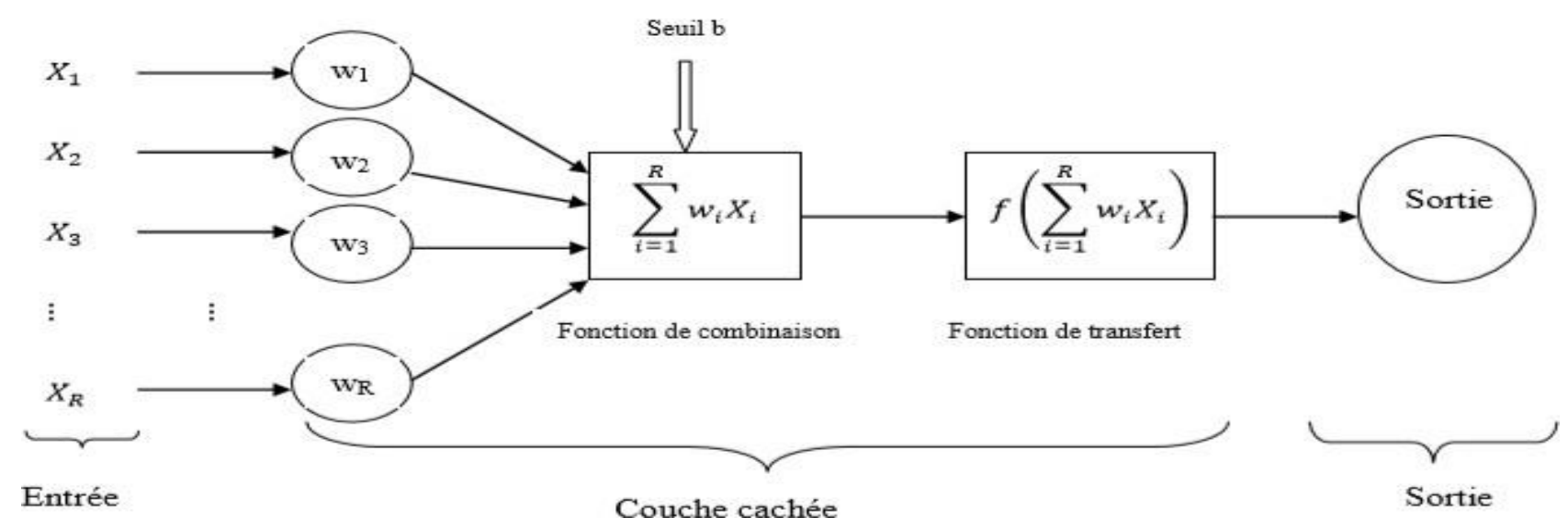

Figure 2. Conception d'un Réseau de Neurones. (Adapté de: Tufféry, 2012)

Le neurone ne traite pas chaque information reçue unilatéralement, mais effectue une somme pondérée de toutes les entrées. Cette somme est représentée sous la fonction de combinaison suivante:

$$
a=\sum_{i=1}^{R} w_{i} x_{i}-b
$$

Avec :

$\mathrm{b}$ : le biais de neurone ou seuil d'activation du neurone.

$\mathrm{X}_{\mathrm{i}}$ : l'information qui parvient aux neurones de rang i de la couche d'entrée

${ }^{6}$ Jantzen. J (1998), “Introduction to perceptron Networks “, report no 98-H 873 (nnet), 25 Oct, Denmark, page27. 
$\mathrm{R}$ : le nombre d'informations.

$w_{i}$ : la pondération du signal émis par le neurone de la couche d'entrée vers le neurone de la couche cachée.

a : le niveau d'activation du neurone, qui est le signal total reçu par le neurone de la couche cachée.

Il existe différents types de réseaux qu'on peut les distinguer par lu nombre de couches et leurs structures :

- Les réseaux à une seule couche cachée.

- Les réseaux multicouches.

- Les réseaux récurrents

\section{La phase de la fonction de transfert}

Chaque neurone reçoit les informations transformées par les neurones de la couche précédente avec lesquels il se trouve en relation pour calculer son potentiel d'activation. La fonction d'activation, ou fonction de transfert, est la fonction qui détermine l'output d'un neurone à partir de la somme des poids pondérée des entrées du réseau. La fonction d'activation des couches cachées est souvent non linéaire, ce qui confère au RNA la propriété de non-linéarité.

Il existe plusieurs types de fonctions de transfert, on trouve : la fonction de Heaviside, La fonction linéaire, La fonction sigmoïde exponentielle, mais la fonction qui nous intéresse dans notre étude est la fonction sigmoïde, elle est définie comme une fonction réelle de $\mathrm{R}$ vers continue, bornée, sachant que sa dérivée est toujours positive. Elle peut prendre deux formes : la fonction logistique exponentielle, ou la fonction hyperbolique. Cette fonction est la plus utilisée dans les RNA de type multi perceptron : La fonction hyperbolique tangente, (Masters (1993)).

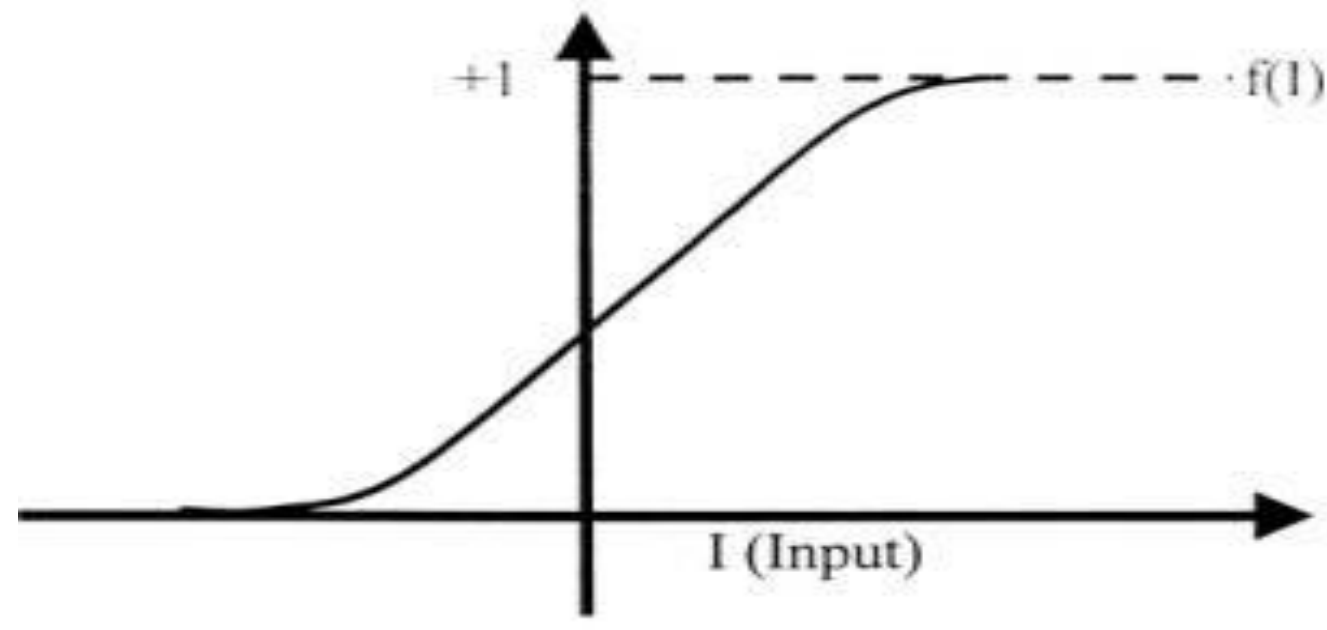

\section{Sigmoid function}

Figure 3. Fonction de transfert (La fonction sigmoïde)

Source: Park S. W, Hwang Y. G, Kang B .C, Yeo S. W, (2001), "Total handle evaluation from selected mechanical properties of knitted fabrics using neural network", International Journal of Clothing Science and Technology, Vol. 13 Iss: 2, pp.106-114 
Cette fonction est définie par la formule suivante :

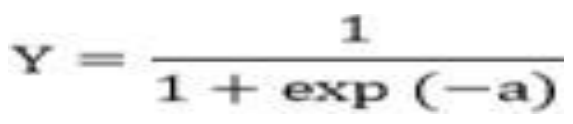

Avec Y comprise entre 0 et 1.

\section{La phase d'apprntissage}

Parmi les caractéristiques les plus importantes des réseaux de neurones est l'apprentissage. Cette dernière repose sur le développement du réseau de neurones jusqu'à l'obtention du résultat attendu. Autrement dit, le fonctionnement du réseau de neurone repose sur la fourniture des données en entrée que le neurone doit apprendre à reconnaître dans le but de les classer en sous-groupes homogènes, c'est ce qu'on appelle : apprentissage non supervisé à des fins descriptives, ou pour les associer à une variable indicatrice de sortie : apprentissage supervisé à des fins prédictives ${ }^{7}$, ce qui convient aux objectifs de notre étude.

On distingue ainsi deux types d'apprentissage : Apprentissage supervisé (Supervised Learning) et Apprentissage non supervisé (Unsupervised Learning). Dans l'apprentissage supervisé, le réseau s'adapte par comparaison entre le résultat qu'il a calculé, en fonction des entrées fournies, et la réponse attendue en sortie. Le système ajuste les données jusqu'à ce qu'il obtienne la bonne sortie.

En ce qui concerne l'apprentissage non supervisé, il est capable de découvrir la forme à partir des données fournies vu que le réseau dans ce cas, doit chercher les ressemblances entre les informations de la base de données, dans ce type d'apprentissage, les données d'entrées existent, mais il n'y a aucune information sur la sortie désirée.

De toutes ces techniques de scoring, on peut assister à des avantages et des limites propres à chaque méthode. Le tableau suivant présente les différentes méthodes de scoring ainsi que leurs avantages et leurs inconvénients:

TABLE I. TABLEAU RÉCAPITULATIF DES TECHNIQUES DE SCORING

\begin{tabular}{|c|c|c|c|}
\hline$\frac{\text { Technique de }}{\text { scoring }}$ & $\underline{\text { Avantages }}$ & Inconvénients & $\underline{\text { Règle classification }}$ \\
\hline \multirow{5}{*}{$\underset{\text { discriminante }}{\stackrel{\text { Analyse }}{\underline{n^{2}}}}$} & Des prédictions explicites. & $\begin{array}{c}\text { Variables explicatives continues et sans } \\
\text { valeurs manquantes. }\end{array}$ & \multirow{5}{*}{$\begin{array}{l}\text { Score d'appartenance } \\
\text { à une classe. }\end{array}$} \\
\hline & Un résultat analytique direct. & Sensible aux individus hors norme. & \\
\hline & Des calculs très rapides & $\begin{array}{l}\text { Absence de tests statistiques de } \\
\text { significativités des coefficients. }\end{array}$ & \\
\hline & $\begin{array}{l}\text { Ne nécessite pas un échantillon de grande taille } \\
\text { pour l'apprentissage. }\end{array}$ & & \\
\hline & $\begin{array}{c}\text { Tient compte des variables qualitatives (procédure } \\
\text { DISQUAL) }\end{array}$ & & \\
\hline
\end{tabular}

${ }^{7}$ Fred Ntoutoume Obiang-Ndong, 2007 «Scoring du risque de crédit des PME par la modélisation statistique et l'intelligence artificielle chez l'UMECUDEFS : une application comparative de la régression logistique et des réseaux de neurones ». Mémoire de fin d'études, Université Cheikh Anta Diop - Faculté Des Sciences Économiques Et De Gestion. 


\begin{tabular}{|c|c|c|c|}
\hline \multirow{7}{*}{$\underline{\text { Régression PLS }}$} & $\begin{array}{c}\text { Utilisable en présence de multi colinéarité entre } \\
\text { les variables. }\end{array}$ & $\begin{array}{l}\text { Nécessité d'adapter au cas d'une réponse } \\
\text { binaire -PLS-DA ou logistique PLS }\end{array}$ & \multirow{7}{*}{$\begin{array}{l}\text { Score d'appartenance } \\
\text { à une classe }\end{array}$} \\
\hline & $\begin{array}{l}\text { Le nombre de variables peut être supérieur au } \\
\text { nombre d'observations. }\end{array}$ & $\begin{array}{c}\text { Calculs supplémentaires (Bootstrap, } \\
\text { validation croisée) pour obtenir des erreurs } \\
\text { standards sur les coefficients. } \\
\end{array}$ & \\
\hline & L'algorithme de la régression PLS est rapide. & & \\
\hline & $\begin{array}{c}\text { L'algorithme de la régression PLS est une suite de } \\
\text { régressions simples sans inversion, ni } \\
\text { diagonalisation de matrices. }\end{array}$ & & \\
\hline & $\begin{array}{l}\text { La prédiction est meilleure avec une régression } \\
\text { PLS sur } \mathrm{p} \text { composantes qu'une régression sur les } \mathrm{p} \\
\text { premières composantes principales. } \\
\end{array}$ & & \\
\hline & Efficace sur un grand volume de données. & & \\
\hline & Possibilité de présence de valeurs manquantes. & & \\
\hline \multirow{6}{*}{$\frac{\text { Régression }}{\underline{\text { logistique }}}$} & $\begin{array}{l}\text { Variables explicatives discrètes, qualitatives ou } \\
\text { continues. }\end{array}$ & $\begin{array}{l}\text { Les variables explicatives doivent être non } \\
\text { colinéaires. }\end{array}$ & \multirow{6}{*}{$\begin{array}{l}\text { Probabilité que } \\
\text { l'évènement de défaut } \\
\text { se produise }\end{array}$} \\
\hline & Variables à expliquer ordinale ou nominale. & $\begin{array}{l}\text { Calcul itératif plus long qu'une analyse } \\
\text { discriminante de Fisher. }\end{array}$ & \\
\hline & $\begin{array}{c}\text { Pas d'hypothèses de multi normalités, ni } \\
\text { d'homoscédasticités pour les variables } \\
\text { explicatives. }\end{array}$ & $\begin{array}{l}\text { La précision est moindre que celle de } \\
\text { l'analyse discriminante. }\end{array}$ & \\
\hline & $\begin{array}{l}\text { Possibilité de prise en compte les interactions } \\
\text { entre variables. }\end{array}$ & $\begin{array}{l}\text { La régression logistique ne converge pas } \\
\text { toujours vers une solution optimale. }\end{array}$ & \\
\hline & \multirow[t]{2}{*}{ Résultats faciles à interpréter. } & $\mathrm{Ne}$ traite pas les valeurs manquantes. & \\
\hline & & Sensible aux valeurs hors norme & \\
\hline \multirow{4}{*}{$\frac{\text { Réseaux de }}{\underline{\text { neurones }}}$} & $\begin{array}{l}\text { Modéliser des relations non linéaires entre les } \\
\text { données. }\end{array}$ & $\begin{array}{l}\text { Les résultats ne sont pas explicites et sont } \\
\text { difficile à comprendre par les utilisateurs }\end{array}$ & \multirow{4}{*}{$\begin{array}{c}\text { Affecter } \\
\text { l'appartenance des } \\
\text { individus aux classes } \\
\text { définies }\end{array}$} \\
\hline & Modéliser des problèmes de différents types. & Le risque de sur-apprentissage. & \\
\hline & \multirow{2}{*}{ Résiste aux données défectueuses. } & Ne traite pas un grand nombre de variables. & \\
\hline & & $\begin{array}{l}\text { La convergence vers la meilleure solution } \\
\text { globale n'est pas toujours garantie }\end{array}$ & \\
\hline \multirow{7}{*}{$\frac{\text { Arbres de }}{\underline{\text { décision }}}$} & $\begin{array}{l}\text { Les résultats sont exprimés sous forme de } \\
\text { condition explicites sur les variables d'origine. }\end{array}$ & $\begin{array}{l}\text { La détermination des nœuds du niveau }(\mathrm{n}+1) \\
\text { dépend fortement du nœud précédent }(\mathrm{n}) \text {. }\end{array}$ & \multirow{7}{*}{$\begin{array}{c}\text { Associer une } \\
\text { observation à } \\
\text { l'attribut attaché à la } \\
\text { feuille à laquelle il } \\
\text { appartient. }\end{array}$} \\
\hline & $\begin{array}{c}\text { Compréhensibilité des résultats pour les } \\
\text { utilisateurs. }\end{array}$ & $\begin{array}{l}\text { L'apprentissage d'un arbre de décision } \\
\text { nécessite un nombre assez grand d'individus. }\end{array}$ & \\
\hline & $\begin{array}{l}\text { Les variables explicatives peuvent ne pas suivre } \\
\text { des lois probabilistes particulières }\end{array}$ & $\begin{array}{l}\text { Le score d'un individu dépend de la feuille à } \\
\text { laquelle le conduisent les valeurs de ses } \\
\text { prédicteurs. }\end{array}$ & \\
\hline & $\begin{array}{c}\text { Les arbres ne sont pas affectés par les individus } \\
\text { hors norme. }\end{array}$ & & \\
\hline & Traite les données manquantes. & & \\
\hline & $\begin{array}{c}\text { Tous types de variables : continues, discrètes et } \\
\text { qualitatives. }\end{array}$ & & \\
\hline & Simple à utiliser. & & \\
\hline \multirow{5}{*}{$\frac{\text { Support Vector }}{\underline{\text { Machine }}}$} & $\begin{array}{l}\text { Capacités à modéliser les phénomènes non } \\
\text { linéaires. }\end{array}$ & Résultats non explicites. & \multirow{5}{*}{$\begin{array}{l}\text { Estimation d'une } \\
\text { frontière de } \\
\text { classification et } \\
\text { l'affectation d'un } \\
\text { individu à une classe } \\
\text { se fait par rapport à sa } \\
\text { position à cette } \\
\text { frontière. } \\
\end{array}$} \\
\hline & Précision de prédictions dans certains cas. & Difficulté des choix des paramètres. & \\
\hline & & Temps de calcul longs & \\
\hline & & Risque de sur-apprentissage. & \\
\hline & & Programmable sur peu de logiciels. & \\
\hline \multirow{5}{*}{$\frac{\text { Algorithmes }}{\text { génétiques }}$} & \multirow{5}{*}{$\begin{array}{l}\text { Améliorer la performance de certains outils de } \\
\text { prédiction comme les réseaux de neurones. }\end{array}$} & Algorithme assez lent. & \multirow{5}{*}{$\begin{array}{l}\text { Trouver des règles de } \\
\text { prédictions } \\
\text { intéressantes pour les } \\
\text { appliquées aux } \\
\text { individus et trouver } \\
\text { l'attribut qui leur } \\
\text { correspond. }\end{array}$} \\
\hline & & $\begin{array}{l}\text { La complexité de cet algorithme augmente de } \\
\text { manière exponentielle en fonction du nombre } \\
\text { de règles utilisées }\end{array}$ & \\
\hline & & $\begin{array}{l}\text { Utilisable sur un volume de données assez } \\
\text { faibles. }\end{array}$ & \\
\hline & & Réglage délicat. & \\
\hline & & Peu répondu dans les logiciels. & \\
\hline
\end{tabular}




\section{CONCLUSION}

Les publications les plus connues dans le domaine de l'intelligence artificielle sont celles de Coats et Fant, (1993), Zang, Hu, Pattuwo et Indro, (1999), qui ont démontré que les réseaux neurones sont plus performants que les autres méthodes traditionnelles de prédiction du phénomène du risque de défaillance, ces réseaux de neurones permettent d'économiser le temps pour les analystes financiers et d'attribuer une flexibilité d'intégration de nouvelles règles.

Dans le cadre de la prédiction du risque de non-remboursement de crédit, une analyse comparative a été effectué par Malhotra (2002), entre l'approche neuronal et l'analyse discriminante, selon les résultats obtenus, il a conclu que la probabilité du défaut est mieux identifiée par les réseaux de neurones que par l'analyse discriminante.

Dans leur étude de prévision du risque de crédit, Younés Boujelbene, Sihem Khemakhem, (2013), ont analysé un échantillon composé de 86 entreprises tunisiennes et une batterie de 15 ratios financiers, ils ont conclu que la technique "neuronale" est meilleure en termes de prévisibilité que les méthodes traditionnelles.

De même, Charalambous et al., (2000), ont réalisé une étude comparative entre la régression logistique et les différents types de réseau de neurones particulièrement le perceptron multicouche, les cartes de Kohonen, la fonction RBC. Ils ont conclu que les méthodes contemporaines du réseau de neurones donnent des résultats supérieurs à ceux obtenus par le modèle Logit.

\section{BIBLIOGRAPHIE}

[1] Abdelwahed OMRI, Meryem BELLOUMA. (2008). «L'impact de la qualité de la Relation Banque-Entreprise sur la prime de risque exigée des entreprises tunisiennes », La Revue des Sciences de Gestion 2008/1 (n²29), p. 95-102.

[2] Agnès Bédué, Nathalie Lévy. (1997). «Relation banque-entreprise et coût du crédit. In: Revue d'économie financière, n³9, Réflexion sur le système bancaire français. » pp. 179- 200.

[3] Asma GUIZANI. (2015). Traitement des dossiers refusés dans le processus d'octroi de crédit aux particuliers. Thèse de doctorat en sciences de gestion.

[4] Aubier Maud, Cherbonnier Frédéric. (2007). « L'accès des entreprises au crédit bancaire » n 177, p.121-128.

[5] Bassolé, L., 2006, "Responsabilité conjointe et performance des groupes de crédit", In le développement face à la pauvreté: Réseau analyse économique et développement,

[6] Economica, 329p.

[7] Dagobert Ngongang. (2015). « Asymétrie d'information et rationnement du crédit bancaire dans les P.M.E. camerounaises », p: 11

[8] Darsa, Jean-David. (2010). « La gestion du risque crédit client: Identifier, comprendre, maîtriser. » Edition: Gereso.

[9] Éric Séverin. (2005). «Financement des Entreprises Avantages et Inconvénients de la Relation Bancaire », Revue Banque N ${ }^{\circ}$ 673.

[10] Kharoubi, Cécile, Thomas, Philippe. (2016). « Analyse du risque de crédit : Banque \& Marchés ». Edition 2, Revue Banque

[11] Michel Dietsch, Valérie Golitin Boubakari. (2002). «L'évolution des relations banques- entreprises dans les années 1990 ». Bulletin de la commission bancaire. $N^{\circ} 27-$ Novembre 2002 P: 41.

[12] Maria Psillaki. (1995). « Rationnement du crédit et PME: une tentative de mise en relation », Revue internationale P.M.E. Volume 8, numéro 3-4, 1995/p: 69

[13] Michel DIETSCH, Valérie Golitin BOUBAKARI. (2002). «L'évolution des relations banques-entreprises dans les années $1990 »$. Bulletin de la commission bancaire. N²7- Novembre 2002 P: 41. 
[14] Michel DIETSCH, Joël PETEY. (2003). « Mesure et gestion du risque de crédit dans les institutions financières. » Revue Banque.

[15] Mohammed LEGYSSER. (2011). «La relation Banque-Entreprise: les résultats d'une enquête auprès d'un échantillon de PME de la région orientale. » p: 170. Revue Marocaine d'administration locale et de développement 2011. Num: 97-98.

[16] HAFSA YERROU. (2018). « Le risque du crédit bancaire: Revue de littérature sur les règles bâloises et l'entrée en vigueur de l'IFRS 9 » p: 313. Revue du contrôle de la comptabilité et de l'audit 2018. Num: 7

[17] Nicolas Eber. (2001). «Les relations bancaires de long terme, une revue de la littérature, Revue d'économie politique. » 2001/2 (Vol. 111) Pages : 136 DOI : 10.3917/redp.112.0195 Éditeur : Dalloz

[18] Rim BOUSSAADA. (2012). «L'impact de la gouvernance Bancaire et de la Relation Bancaire sur le risque de crédit: Cas des Banques Tunisiennes ». Doctorat en sciences de gestion, soutenue le 14 Septembre 2012.

[19] Statnik J.-C. (1997). « Asymétrie d'information et rationnement partiel du crédit ». Thèse de doctorat, Université Lille II.

[20] Stiglitz, J.E., 1993, "The role of the state in Financial Markets". Proceedings of the World Bank Annual Conference on Economic Development, Washington D.C. International Bank for Reconstruction and Development/World Bank, pp. 19-5 